\title{
Lactoperoxidase, an Antimicrobial Milk Protein, as a Potential Activator of Carcinogenic Heterocyclic Amines in Breast Cancer
}

\author{
ISHFAQ AHMAD SHEIKH ${ }^{1}$, ESSAM HUSSAIN JIFFRI ${ }^{2}$, MOHAMMAD AMJAD KAMAL ${ }^{1}$, \\ GHULAM MD ASHRAF ${ }^{1}$ and MOHD AMIN BEG ${ }^{1}$ \\ ${ }^{1}$ King Fahd Medical Research Center, King Abdulaziz University, Jeddah, Kingdom of Saudi Arabia; \\ ${ }^{2}$ Faculty of Applied Medical Sciences, King Abdulaziz University, Jeddah, Kingdom of Saudi Arabia
}

\begin{abstract}
Background: Lactoperoxidase (LPO) is an antimicrobial protein secreted from mammary, salivary and other mucosal glands. It is an important member of heme peroxidase enzymes and the primary peroxidase enzyme present in breast tissues. In addition to the antimicrobial properties, LPO has been shown to be associated with breast cancer etiology. Heterocyclic amines, an important class of environmental and dietary carcinogens, have been increasingly associated with breast cancer etiology. Heterocyclic amines undergo activation in breast tissue as a result of oxidation by LPO. The current study includes three important heterocyclic amines, 2-amino-3-methylimidazo[4,5-f]quinoline (IQ), 2amino-3,8-dimethylimidazo[4,5-f]quinoxaline (MeIQx) and 2amino-1-methy-6-phenylimidazo[4,5-b]-pyridine (PhIP), that have carcinogenic activity. Materials and Methods: The structural binding characterization of IQ, MeIQx and PhIP with LPO was done using in silico approaches. Their binding pattern and interactions with LPO amino acid residues were analyzed. Results: The three compounds bound in the distal heme cavity of LPO without replacing the important water molecule required for oxidation of substrate compounds. PhIP displayed lesser binding affinity for LPO in comparison to IQ and MeIQx. The binding mode of heterocyclic amines in distal heme cavity of LPO resembled to that of substrate binding pattern. Conclusion: The three heterocyclic amines are suggested to act as LPO substrate. The undisturbed water molecule present in distal heme cavity of the LPO is expected to facilitate the oxidation and activation of the three heterocyclic amines. These activated compounds may potentially bind with DNA
\end{abstract}

Correspondence to: Ishfaq Ahmad Sheikh, King Fahd Medical Research Center, King Abdulaziz University, P.O. Box-80216, Jeddah-21589, Kingdom of Saudi Arabia. Tel: +966 532972254, e-mail: iasheikh@kau.edu.sa

Key Words: Heterocyclic amines, IQ, MeIQx, PhIP, breast cancer, lactoperoxidase. in breast tissues forming DNA adducts and may subsequently lead to breast cancer initiation.

Lactoperoxidase (LPO) is a heme-containing enzyme categorized as a member of mammalian peroxidase superfamily (1). It is a $80-\mathrm{kDa}$, glycosylated protein (2-4) consisting of 595 amino acid residues organized into $20 \alpha$ helices and 2 antiparallel $\beta$-strands (5). LPO is a green color protein present in saliva, mucosa, milk etc. and has antimicrobial activity (6-8).

In addition to the antimicrobial functions, LPO has been suggested to play a role in the etiology of breast cancer (9). Breast cancer remains a significant health problem with a deadly outcome in women despite recent medical advancements. According to International Agency for Research on Cancer, breast cancer is the fifth common cause of cancer worldwide (10). In women, it is the most abundant cancer and the second highest cause of cancer-related deaths (11). A report on breast cancer incidence and mortality by Centers for Disease Control and Prevention, Atlanta, GA, USA (12) indicated that breast cancer is the most common cancer in women across all ethnicities in the United States and also the most common cause of cancer-related deaths in Hispanic women. In 2013, 2,109 women died due to breast cancer in the United States (12). Breast cancer is a multifactorial disease and the important factors increasingly implicated in breast cancer include environmental and food carcinogens (13).

Heterocyclic amines constitute an important class of potential food mammary carcinogens and are produced in animal origin foods, especially muscle meat, when amino acids, sugars and creatinine react at high temperatures (14). Heterocyclic amines, thus formed in hyper-cooked and charred meat, when ingested become mutagenic in the body and increase the risk of cancer. In order to become mutagenic, heterocyclic amines undergo a process of bioactivation in human and animal systems by specific oxidizing enzymes, such as oxidases and peroxidases, and are converted into reactive compounds $(9,15)$. LPO has been shown to be involved in oxidation and activation of carcinogenic and 
Table I. Nomenclature, commonly used abbreviations and PubChem IDs of the three heterocyclic amine compounds.

\begin{tabular}{lccc}
\hline S.No & Name & Abbreviation & PubChem ID \\
\hline 1 & 2-amino-3-methylimidazo[4,5-f]quinoline & IQ & 53462 \\
2 & 2-amino-3,8-dimethylimidazo[4,5-f]quinoxaline & MeIQx & 62275 \\
3 & 2-amino-1-methy-6-phenylimidazo[4,5-b]pyridine & PhIP & 1530 \\
\hline
\end{tabular}

mutagenic chemical compounds, including heterocyclic amines in the human mammary gland, converting them into highly reactive compounds forming DNA adducts (16-18).

Experimental studies have shown that rats and mice fed with foods containing heterocyclic amines develop tumors in many organs, including mammary carcinomas $(19,20)$. The human population is exposed to heterocyclic amines on daily basis and one of the main source is protein-rich food items, such as meat and fish, which are smoked or cooked for longer time, especially grilling, broiling and pan-frying above $300^{\circ} \mathrm{C}(14-15,21-22)$. Epidemiological studies on the association of exposure to heterocyclic amines in food and risk of developing various cancers, including breast cancer, have been reviewed (23). Heterocyclic amine exposure through consumption of well-done meat was positively associated with breast cancer in women in Uruguay (24). In another study, a highly significant dose-response relationship was found between breast cancer risk and meat doneness score in postmenopausal women in Iowa, USA $(21,25)$. A five-fold higher breast cancer risk was found in women who regularly consumed fully cooked meat.

The most abundant heterocyclic amines in cooked meat are 2-amino-3-methylimidazo[4,5-f]quinoline (IQ), 2-amino3,8-dimethylimidazo (4,5-f) quinoxaline (MeIQx) and 2amino-1-methyl-6-phenylimidazo (4,5-b) pyridine (PhIP), $(14,26)$. LPO has been shown to catalyze the oxidation of the three mutagenic heterocyclic amines and form DNA adducts $(9,17)$. However, studies describing the binding affinity and binding pattern of these heterocyclic amines into the distal heme cavity of LPO are apparently not available.

Herein, we present and discuss the structural binding characterization of LPO with the above mentioned three important heterocyclic amines, IQ and MeIQx and PhIP, using the Schrödinger Induced Fit Docking (IFD) approach. The structural aspects, amino acid residue interactions, binding affinity and binding mode of the three heterocyclic amines are described.

\section{Materials and Methods}

Schrödinger 2015 suite (Schrödinger; LLC, New York, NY, USA) was used for in silico docking studies of the three heterocyclic amines IQ and MeIQx and PhIP with LPO, with a methodology being given previously in detail (27).
Data retrieval. PubChem compound database (https:// pubchem.ncbi.nlm.nih.gov/) was used for retrieving the threedimensional structures of PhIP, IQ and MeIQx. The twodimensional structures of the three heterocyclic amines are presented (Figure 1) and their PubChem compound identities (CIDs) along with abbreviations are also provided (Table I).

Protein selection and preparation. The three-dimensional structure of bubaline homolog of LPO co-complexed with thiocyanate was obtained from the Protein Data Bank (PDB) (http://www.rcsb.org) and has a resolution of $2.4 \AA$ (PDB code: 3ERH). Bubaline LPO has $76 \%$ similarity with human LPO. This PDB complex structure was developed using preparation wizard workflow of Schrödinger as described in detail previously (27).

Ligand preparation. Ligands were prepared using LigPrep module (LigPrep, version 3.1; Schrödinger). The methodology has been described previously (27).

IFD. Prime module of Schrödinger 2015 suite was used for the execution of IFD. The methodology has been described previously (27).

Binding energy calculations. For calculating the binding affinity of IQ and MeIQx and PhIP with LPO, Molecular Mechanics with Generalized Born and Surface Area (MM-GBSA) model present in Prime module of Schrödinger 2015-3 was used.

\section{Results}

Molecular docking. The amino acid residues of LPO interacting with IQ, MeIQx and PhIP are shown (Figure 2). In the LPO-IQ complex, 12 amino acid residues of LPO were involved in interactions with IQ. Whereas, in LPOMeIQx and LPO-PhIP complexes, 13 and 11 residues of LPO were engaged in interaction with MeIQx and PhIP, respectively. Moreover, all three amines, IQ, MeIQx and PhIP, interacted with the heme ring of LPO. When the docking complexes of the three amines with LPO were compared, eight interacting residues (Phe-113, Ala-114, Pro115, Glu-116, Arg-255, Glu-258, Phe-381 and Arg-440) were found to be common. Additionally, in LPO-IQ and LPOMeIQx complexes, three residue interactions (Gln-105, His109 and Leu-262) were common but absent in LPO-PhIP complex. Both IQ and MeIQx displayed a common hydrogen bonding interaction with Ala-114, which, however, was not present in the PhIP complex. 


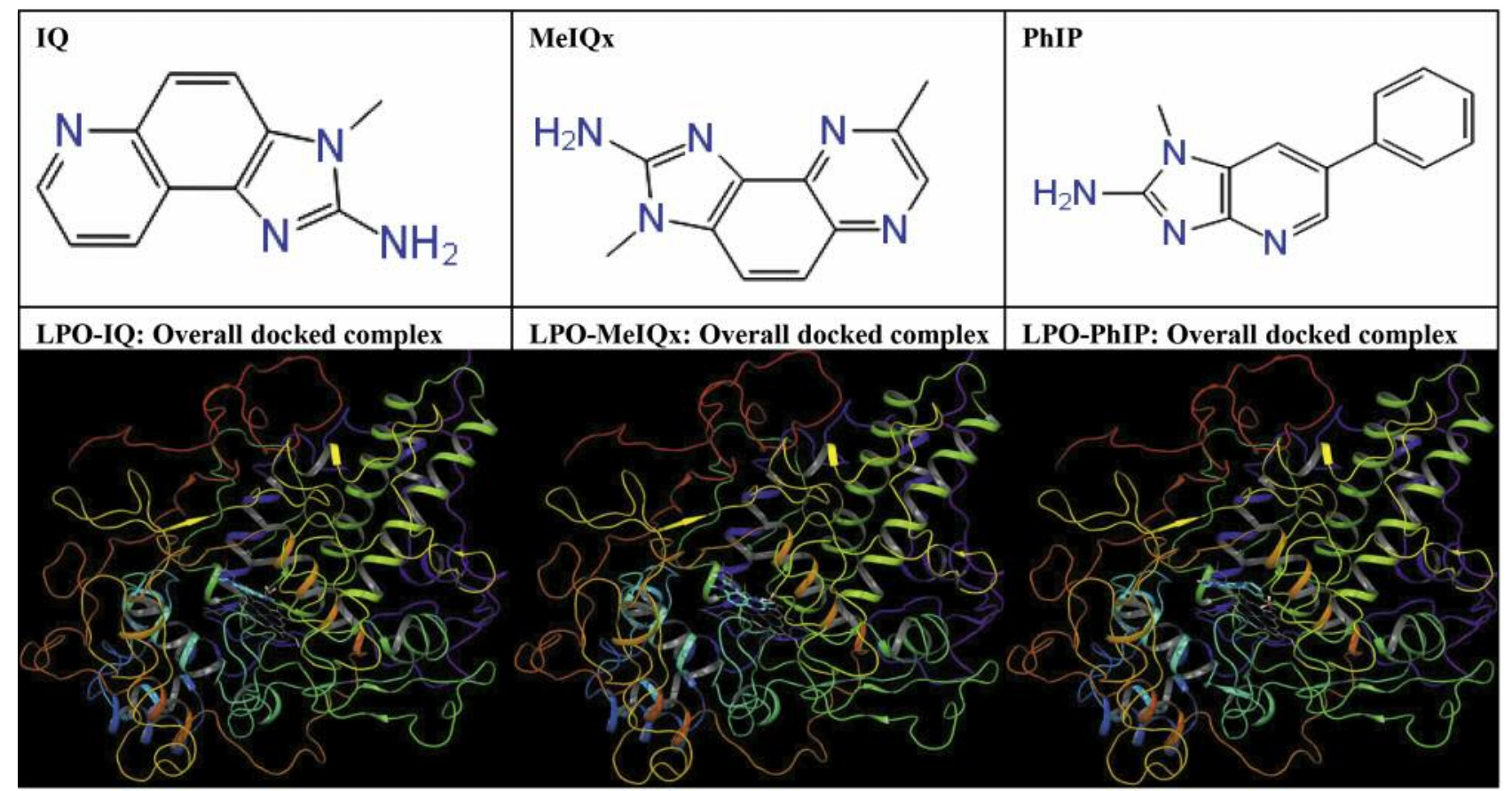

Figure 1. Two-dimensional representation of the three heterocyclic amines 2-amino-3-methylimidazo[4,5-f]quinoline (IQ), 2-amino-3,8dimethylimidazo[4,5-f]quinoxaline (MeIQx) and 2-amino-1-methy-6-phenylimidazo[4,5-b]-pyridine (PhIP) and ribbon form representation of their docked complexes with lactoperoxidase (LPO).

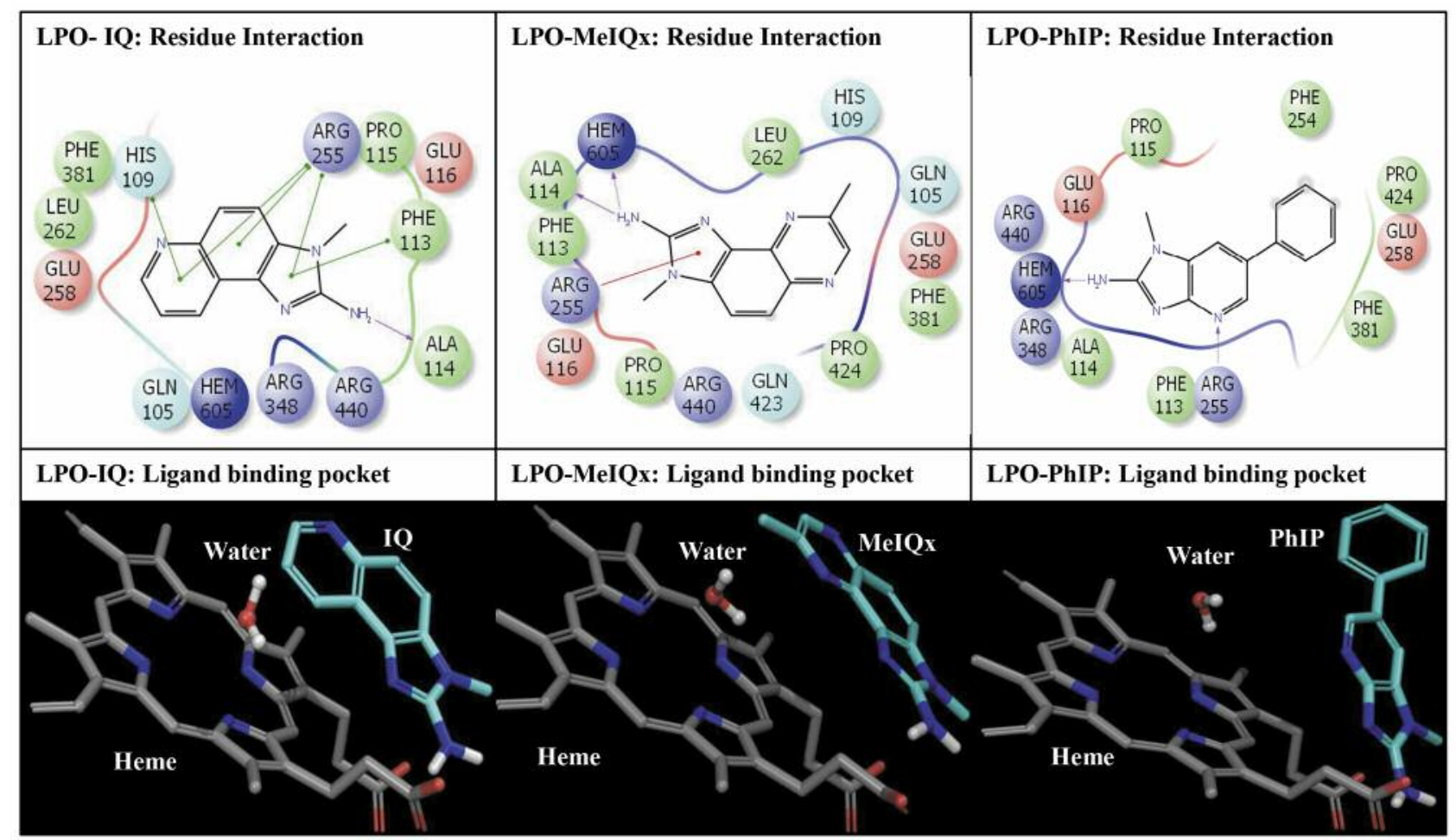

Figure 2. Amino-acid residue interaction of the three heterocyclic amines 2-amino-3-methylimidazo[4,5f]quinoline (IQ), 2-amino-3,8dimethylimidazo[4,5 f]quinoxaline (MeIQx) and 2-amino-1-methy-6-phenylimidazo[4,5-b]-pyridine (PhIP) in the binding pocket of lactoperoxidase (LPO) and their binding pattern in distal heme cavity. 
Table II. Number of interacting residues, dock score, glide score and binding affinity values (Molecular Mechanics with Generalized Born and Surface Area (MM-GBSA) values) of 2-amino-3-methylimidazo[4,5f]quinoline (IQ), 2-amino-3,8-dimethylimidazo[4,5 f]quinoxaline (MeIQx) and 2-amino-1-methy-6-phenylimidazo[4,5-b]-pyridine (PhIP) after IFD with lactoperoxidase (LPO).

\begin{tabular}{lccccc}
\hline Target & Ligand & Number of interacting residues & Docking score $(\mathrm{Kcal} / \mathrm{mol})$ & Glide score (Kcal/mol) & MM-GBSA (Kcal/mol) \\
\hline LPO & IQ & 12 & -6.75 & -6.78 & -66.41 \\
LPO & MeIQx & 13 & -7.37 & -7.37 & -64.50 \\
LPO & PhIP & 11 & -6.59 & -6.70 & -48.11 \\
\hline
\end{tabular}

The docking complex of MeIQx displayed one more hydrogen bonding interaction with the heme ring. Similarly, PhIP exhibited two hydrogen bonding interactions, one with heme and another with Arg-255. In LPO-IQ docking complex, pi-pi interactions with His-109, Phe-113 and Arg255 were also observed. However, only one cation-pi interaction with Arg-255 was displayed by LPO-MeIQx docked complex and no pi-pi interaction was seen in LPOPhIP complex. All three heterocyclic amines bound in the distal heme cavity of LPO at the substrate binding site but did not disturb the important water molecule required for oxidation reaction to occur (Figure 2). Binding affinity values, glide score, as well as dock score for all three ligands, have also been calculated and are presented (Table II).

\section{Discussion}

Heterocyclic amines are generated from protein-rich foods, such as meat and fish cooked at high temperature for long time (28-30) and their formation varies with the type of meat and the kind of approach adopted for cooking $(14,26)$. In women, a strong correlation was observed between dietary heterocyclic amine intake and DNA adducts levels in breast tissues (22). In another study, in New York, higher susceptibility for postmenopausal breast cancer was found in women consuming meat cooked in a manner that promotes formation of carcinogenic heterocyclic amines (29).

Heterocyclic amines display lipophilic property and accumulate in breast adipose tissue, resulting in their susceptibility to activation by peroxidases present locally in breast tissue (31). LPO has been reported to be involved in activation of IQ, MeIQx and PhIP in breast tissue, thus increasing the risk of breast cancer (9).

In the current investigation, three heterocyclic amines, IQ, MeIQx and PhIP, bound in the distal heme cavity of LPO and their binding pattern mimicked the pattern of LPO substrates. As reported previously (32), LPO substrates bind in the distal heme cavity without replacing the conserved water molecule. Conversely, LPO inhibitors replace the conserved water molecule upon binding in the distal heme cavity of LPO (33). This striking property of replacement of conserved water molecule distinguishes LPO substrates from LPO inhibitors. In our study, binding of IQ, MeIQx and PhIP in the substrate binding site did not disturb the conserved water molecule in the distal heme cavity of LPO and, therefore, confirmed their substrate nature. The conserved water molecule is believed to facilitate the activation of the above indicated heterocyclic amines and convert them into reactive products. These reactive products bind with DNA forming DNA adducts and may, subsequently, lead to breast carcinogenesis.

The binding affinity of PhIP for LPO was lower in comparison to that of IQ and MeIQx. The lower binding affinity for PhIP is supported by similar results in an in vitro study previously carried on human breast tissues (9). The lesser binding affinity of PhIP for LPO, in comparison to other two heterocyclic amines, could be attributed to its different placement in the substrate binding pocket. That is, $\mathrm{PhIP}$ was bound in substrate binding pocket little away from the heme ring, whereas IQ and MeIQx were bound much deeper in the distal heme cavity of LPO. Moreover, less number of interactions was formed by PhIP with LPO in comparison to those of IQ and MeIQx. The binding affinity value of IQ for LPO was slightly higher than that of MeIQx for LPO.

\section{Conclusion}

The current investigation showed that the three heterocyclic amines IQ, MeIQx and PhIP were bound with LPO without replacing the important water molecule required for substrate oxidation. The estimated binding affinity of IQ for LPO was slightly higher than that of MeIQx; however, PhIP exhibited comparatively lesser binding affinity values than both IQ and MeIQx. The binding pattern of all three compounds in the distal heme cavity of LPO mimicked that of LPO substrates. Therefore, the three heterocyclic amines IQ and MeIQx and PhIP, acting as LPO substrates, may potentially become activated into reactive compounds. These compounds may form mutagenic DNA adducts in mammary tissues and, eventually, behave as potential carcinogens that may lead to breast cancer in women. 


\section{Conflicts of Interest}

The Authors declare that no competing interests exist.

\section{Acknowledgements}

This project was funded by the National Plan for Science, Technology and Innovation (MAARIFAH) - King Abdulaziz City for Science and Technology - the Kingdom of Saudi Arabia - award number (12-BIO3082-03). The Authors also acknowledge the Science and Technology Unit, King Abdulaziz University, for their technical support.

\section{References}

1 Nichol AW, Angel LA, Moon T and Clezy PS: Lactoperoxidase haem, an iron porphyrin thiol. Biochem J 247(1): 147-150, 1987.

2 Thomas EL, Bozeman PM and Leam DB: Lactoperoxidase: Structure and catalytic properties. In: Reverse J, Everse KI and Graham MB (eds.). Peroxidases in Chemistry and Biology, Vol I. CRC Press, Boca Raton, FL, pp. 123-142, 1991.

3 Chang CS, Sinclair R, Khalid S, Yamazaki I, Nakamura S and Powers L: An extended X-ray absorption fine structure investigation of the structure of the active site of lactoperoxidase. Biochemistry 32: 2780-2786, 1993.

4 Ferrari RP, Laurenti E, Cecchini PI, Gambino $O$ and Sondergaard I: Spectroscopic investigations on the highly purified lactoperoxidase Fe(III)- heme catalytic site. J Inorg Biochem 58: 109-127, 1995 .

5 Singh AK, Singh N, Sharma S, Singh SB, Kaur P, Bhushan A, Srinivasan A and Singh TP: Crystal structure of lactoperoxidase at $2.4 \AA$ resolution. J Mol Biol 376(4): 1060-1075, 2008.

6 Ahariz M and Courtois P: Candida albicans susceptibility to lactoperoxidase-generated hypoiodite. Clin Cosmet and Investig Dent 2: 69-78, 2010

7 Welk A, Meller C, Schubert R, Schwahn C, Kramer A and Below $\mathrm{H}$ : Effect of lactoperoxidase on the antimicrobial effectiveness of the thiocyanate hydrogen peroxide combination in a quantitative suspension test. BMC Microbiology 9(134): 1-8, 2009.

8 Pruitt KM and Tenovou JO: The Lactoperoxidase System. Chemistry and biological significance. Marcel Dekker, New York, USA, pp. 257, 1985.

9 Gorlewska-Roberts KM, Teitel CH, Lay JO Jr, Roberts DW and Kadlubar FF: Lactoperoxidase-catalyzed activation of carcinogenic aromatic and heterocyclic amines. Chem Res Toxicol 17(12): 1659-1666, 2004

10 World Health Organization IARC Breast Cancer. Estimates Incidence, Mortality and Prevalence Worldwide in 2012. Available at: http://globocan.iarc.fr/Pages/fact_ sheets_ cancer.aspx. Last accessed: September 2016.

11 Tracy JC, Mildenhall NR, Wein RO and O'Leary MA: Breast cancer metastases to the head and neck: Case series and literature review. Ear Nose Throat J 96(3): E21-E24, 2017.

12 U.S. Cancer Statistics Working Group. United States Cancer Statistics: 1999-2013 Incidence and Mortality Web-based Report. Atlanta (GA): Department of Health and Human Services, Centers for Disease Control and Prevention, and National Cancer Institute; 2016. Available at: http://www.cdc.gov/usc.
13 Henderson IC: Risk factors for breast cancer development. Cancer 71: 2127-2140, 1993.

14 Cross AJ and Sinha R: Meat-related mutagens/carcinogens in the etiology of colorectal cancer. Environ Mol Mutagen 44(1): 44$55,2004$.

15 Jagerstad M and Skog K: Genotoxicity of heat-processed foods. Mutat Res 574(1-2): 156-172, 2005.

16 Josephy PD: The role of peroxidase-catalyzed activation of aromatic amines in breast cancer. Mutagenesis 11(1): 3-7, 1996.

17 Williams JA, Stone EM, Millar BC, Hewer A and Phillips DH: Pathways of heterocyclic amine activation in the breast: DNA adducts of 2-amino-3-methylimidazo[4,5-f]quinoline (IQ) formed by peroxidases and in human mammary epithelial cells and fibroblasts. Mutagenesis 15(2): 149-154, 2000.

18 Ghibaudi EM, Laurenti E, Beltramo P and Ferrari RP: Can estrogenic radicals, generated by lactoperoxidase, be involved in the molecular mechanism of breast carcinogenesis? Redox Rep 5(4): 229-35, 2000.

19 Kato T, Migita H, Ohgaki H, sato S, Takayama S and Sugimura T: Induction of tumors in the Zymbal gland, oral cavity, colon, skin and mammary gland of F344 rats by a mutagenic compound, 2-amino-3,4-dimethylimidazo[4,5-f]quinoline. Carcinogenesis 10(3): 601-603, 1989.

20 Ito N, Hasegawa R, Sano M, Tamano S, Esumi H, Takayama S and Sugimura T: A new colon and mammary carcinogen in cooked food, 2-amino-1-methyl-6-phenyhmidazo[4-fc]pyridine (PhIP). Carcinogenesis 12: 1503-1506, 1991.

21 Sinha R, Gustafson DR, Kulldorff M, Wen WQ, Cerhan JR and Zheng W: 2-amino-1-methyl-6-phenylimidazo[4,5-b]pyridine, a carcinogen in high-temperature-cooked meat, and breast cancer risk. J National Cancer Inst 92(16): 1352-1354, 2000.

22 Rohrmann S, Lukas Jung SU, Linseisen J and Pfau W: Dietary intake of meat and meat-derived heterocyclic aromatic amines and their correlation with DNA adducts in female breast tissue. Mutagenesis 24(2): 127-132, 2009.

23 Zheng W and Lee SA: Well-done Meat Intake, Heterocyclic Amine Exposure, and Cancer Risk. Nutr Cancer 61(4): 437-446, 2009.

24 DeStefani E, Ronco A, Mendilaharsu M, Guidobono M and Deneo-Pellegrini $\mathrm{H}$ : Meat intake, heterocyclic amines, and risk of breast cancer: A case-control study in Uruguay. Cancer Epidemiol Biomark and Prev 6(8): 573-581, 1997.

25 Zheng W, Gustafson DR, Sinha R, Cerhan JR, Moore D, Hong CP, Anderson KE, Kushi LH, Sellers TA and Folsom AR: Welldone meat intake and risk of breast cancer. J Natl Cancer Inst 90(22): 1724-1729, 1998.

26 Knize MG and Felton JS: Formation and human risk of carcinogenic heterocyclic amines formed from natural precursors in meat. Nutrition Rev 63(5): 158-165, 2005.

27 Sheikh IA: Stereoselectivity and potential endocrine disrupting activity of Bis-(2-ethylhexyl)phthalate (DEHP) against human progesterone receptor: A computational perspective. J Appl Toxicol 36(5): 741-747, 2016.

28 Snyderwine EG: Mammary gland carcinogenesis by food-derived heterocyclic amines: Metabolism and additional factors influencing carcinogenesis by 2-amino-1-methyl-6-phenylimidazo[4,5b]pyridine (PhIP). Environ Mol Mutagen 39(2-3): 165-170, 2002.

29 Steck SE, Gaudet MM, Eng SM, Britton JA, Teitelbaum SL, Neugut AI, Santella RM and Gammon MD: Cooked meat and risk of breast cancer. Lifetime versus recent dietary intake. Epidemiology 18(3): 373-382, 2007. 
30 Lauber SN and Gooderham NJ: The cooked meat-derived mammary carcinogen 2-amino-1-methyl-6-phenylimidazo[4,5b]pyridine promotes invasive behaviour of breast cancer cells. Toxicology 279(1-3): 139-145, 2011.

31 Beer AE and Billingham RE: Adipose tissue, a neglected factor in aetiology of breast cancer. Lancet 2(8084): 296, 1978.

32 Sheikh IA, Singh AK, SinghN, Sinha M, Singh SB, Bhushan A, Kaur P, Srinivasan A, Sharma S and Singh TP: Structural evidence of substrate specificity in mammalian peroxidases: Structure of the thiocyanate complex with lactoperoxidase and its interactions at $2.4 \AA$ resolution. J Biol Chem 284(22): 1484914856, 2009.
33 Singh AK, Singh N, Sinha M, Bhushan A, Kaur P, Srinivasan A, Sharma $\mathrm{S}$ and Singh TP: Binding modes of aromatic ligands to mammalian heme peroxidases with associated functional implications: Crystal structures of lactoperoxidase complexes with acetylsalicylic acid, salicylhydroxamic acid, and benzylhydroxamic acid. J Biol Chem 284(30): 20311-20318, 2009. 